\title{
A CRITERION FOR DISCRETE BRANCHING LAWS FOR KLEIN FOUR SYMMETRIC PAIRS AND ITS APPLICATION TO $E_{6(-14)}$
}

\author{
HAIAN HE
}

\begin{abstract}
Let $G$ be a noncompact connected simple Lie group, and $\left(G, G^{\Gamma}\right)$ a Klein four symmetric pair. In this paper, the author shows a necessary condition for the discrete decomposability of unitarizable simple $(\mathfrak{g}, K)$-modules for Klein for symmetric pairs. Precisely, if certain conditions hold for $\left(G, G^{\Gamma}\right)$, there does not exist any unitarizable simple $(\mathfrak{g}, K)$-module that is discretely decomposable as a $\left(\mathfrak{g}^{\Gamma}, K^{\Gamma}\right)$-module. As an application, for $G=\mathrm{E}_{6(-14)}$, the author obtains a complete classification of Klein four symmetric pairs $\left(G, G^{\Gamma}\right)$ with $G^{\Gamma}$ noncompact, such that there exists at least one nontrivial unitarizable simple $(\mathfrak{g}, K)$-module that is discretely decomposable as a $\left(\mathfrak{g}^{\Gamma}, K^{\Gamma}\right)$-module and is also discretely decomposable as a $\left(\mathfrak{g}^{\sigma}, K^{\sigma}\right)$-module for some nonidentity element $\sigma \in \Gamma$.
\end{abstract}

\section{INTRODUCTION}

Let $G$ be a noncompact connected simple Lie group, and $\Gamma$ a Klein four subgroup of the automorphism group Aut $G$ of $G$. Then $\left(G, G^{\Gamma}\right)$ forms a Klein four symmetric pair, where $G^{\Gamma}$ is the subgroup of the fixed points under action of all elements in $\Gamma$ on $G$. Write $\mathfrak{g}$ for the Lie algebra of $G$, and $\mathfrak{g}^{\Gamma}$ for the Lie subalgebra of $\mathfrak{g}$ corresponding to $G^{\Gamma}$. Take a $\Gamma$-stable maximal compact subgroup $K$ of $G$ so that $K^{\Gamma}=K \cap G^{\Gamma}$ is a maximal compact subgroup of $G^{\Gamma}$. For a $(\mathfrak{g}, K)$-module $X$, it can be regarded as a $\left(\mathfrak{g}^{\Gamma}, K^{\Gamma}\right)$-module by restriction. A fundamental problem is to classify all the Klein four symmetric pairs $\left(G, G^{\Gamma}\right)$ such that there exists at least one nontrivial unitarizable simple $(\mathfrak{g}, K)$-module that is discretely decomposable as a $\left(\mathfrak{g}^{\Gamma}, K^{\Gamma}\right)$-module. If $G^{\Gamma}$ is compact, the problem is trivial because any unitarizable simple $(\mathfrak{g}, K)$-module is discretely decomposable as a $\left(\mathfrak{g}^{\Gamma}, K^{\Gamma}\right)$-module. Hence, the author is only interested in the case when $G^{\Gamma}$ is noncompact.

When $G$ is supposed to be an exceptional simple Lie group of Hermitian type, the discretely decomposable restrictions of unitarizable simple $(\mathfrak{g}, K)$-modules for Klein four symmetric pairs ware studied in [1], [2], and [3]. In this case, one may define Klein four symmetric pairs of holomorphic type. The discretely decomposable condition for Klein four symmetric pairs of holomorphic type is easy to deal with because any highest / lowest weight simple $(\mathfrak{g}, K)$-module is discretely decomposable as a $\left(\mathfrak{g}^{\Gamma}, K^{\Gamma}\right)$-module. As for Klein four symmetric pairs of nonholomorphic type, it is difficult. In [3], the author classified the Klein four symmetric pairs $\left(G, G^{\Gamma}\right)$ for exceptional simple Lie groups of Hermitian type such that there exists at least one nontrivial unitarizable simple $(\mathfrak{g}, K)$-module $X$ that is both discretely decomposable as a $\left(\mathfrak{g}^{\Gamma}, K^{\Gamma}\right)$-module and is discretely decomposable as a $\left(\mathfrak{g}^{\sigma}, K^{\sigma}\right)$-module for some nonidentity element $\sigma \in \Gamma$ of anti-holomorphic type. Here, $\mathfrak{g}^{\sigma}:=\{X \in \mathfrak{g} \mid \sigma X=X\}$.

Suppose that $\Gamma$ is generated by the two involutive automorphisms $\sigma$ and $\tau$, and then $\Gamma=$ $\{1, \sigma, \tau, \sigma \tau\}$. The nonidentity elements in $\Gamma$ define three symmetric pairs $\left(G, G^{\sigma}\right),\left(G, G^{\tau}\right)$, and $\left(G, G^{\sigma \tau}\right)$ for $G$, where $G^{\sigma}$ (respectively, $G^{\tau}$, or $G^{\sigma \tau}$ ) is the subgroup of the fixed points under the action of $\sigma$ (respectively, $\tau$, or $\sigma \tau$ ) on $G$. The branching rules for symmetric pairs

2010 Mathematics Subject Classification. 22E46.

Key words and phrases. Klein four symmetric pair; branching law; discretely decomposable; unitarizable simple ( $\mathfrak{g}, K)$-module; associated variety. 
were studied by many mathematicians, among which the classification of symmetric pairs $\left(G, G^{\prime}\right)$ such that there exists at least one nontrivial unitarizable simple $(\mathfrak{g}, K)$-module which is discretely decomposable as a $\left(\mathfrak{g}^{\prime}, K^{\prime}\right)$-module was completely solved in [11, Theorem $5.2 \&$ Corollary 5.8] based on the criteria established in [7], [8], and [9], where $\mathfrak{g}^{\prime}$ is the Lie subalgebra of $\mathfrak{g}$ corresponding to $G^{\prime}$.

Associated varieties are useful tools to study the discrete decomposability of the restrictions of unitarizable simple $(\mathfrak{g}, K)$-modules. Roughly speaking, for a simple Lie group $G$ and its reductive subgroup $G^{\prime}$, if $X$ is a unitarizable simple $(\mathfrak{g}, K)$-module which is discretely decomposable as a $\left(\mathfrak{g}^{\prime}, K^{\prime}\right)$-module, and if $Y$ is a nonzero simple $\left(\mathfrak{g}^{\prime}, K^{\prime}\right)$-submodule of $X$, then the projection of the associated variety of $X$ from $\mathfrak{g}$ to $\mathfrak{g}^{\prime}$ is contained in the associated variety of $Y$. Thus, the associated varieties offer a necessary condition for the discretely decomposable restriction of a unitarizable simple $(\mathfrak{g}, K)$-module. In [11], the authors just made use of associated varieties to find a crucial necessary condition for discretely decomposable restrictions for symmetric pairs. As the natural generalization of symmetric pairs, the author studies Klein four symmetric pairs.

The classification of Klein four symmetric pairs is far from clear as that of symmetric pairs, so it is much hard to study their branching laws. However, the classification of Klein four subgroups of automorphism groups of compact Lie algebras was done in [4], and a more general classification of elementary abelian 2-subgroups of automorphism groups of compact Lie algebras was done in [12]. The author will make use of the classification results to study Klein four symmetric pairs.

The article is organized as follows. After a quick review of associated varieties, noncompact maximal roots, and minimal orbits, the author will recall an important result of discretely decomposable restrictions of unitarizable simple $(\mathfrak{g}, K)$-modules for symmetric pairs in Section 2. Then in Section 3, the author will show the main result in this article, which offers a necessary condition for the existence of unitarizable simple $(\mathfrak{g}, K)$-modules whose restrictions are discretely decomposable restrictions for Klein four symmetric pairs, see Theorem 11 and Corollary 12 below. Finally, the author will apply the main result to $\mathrm{E}_{6(-14)}$, and will solve a branching problem for Klein four symmetric pairs of $\mathrm{E}_{6(-14)}$, which is another important result in this article, see Theorem 25 below, and is the complete answer to the problem raised in [3] for $\mathrm{E}_{6(-14)}$.

The author is supported by National Natural Science Foundation of China (Grant Number: 11901378).

\section{Preliminary}

2.1. Associated varieties and minimal orbits. Let $G$ be a noncompact connected simple Lie group with Lie algebra $\mathfrak{g}$. Fix a Cartan decomposition $\mathfrak{g}=\mathfrak{k}+\mathfrak{p}$, write $\mathfrak{g}_{\mathbb{C}}=\mathfrak{k}_{\mathbb{C}}+\mathfrak{p}_{\mathbb{C}}$ for its complexification, $\mathfrak{g}_{\mathbb{C}}^{*}=\mathfrak{k}_{\mathbb{C}}^{*}+\mathfrak{p}_{\mathbb{C}}^{*}$ for the dual space, and $K$ for the connected subgroup of $G$ with Lie algebra $\mathfrak{k}$. Denote by $K_{\mathbb{C}}$ the subgroup of the inner automorphism group Intg $\mathfrak{g}_{\mathbb{C}}$ of $\mathfrak{g}_{\mathbb{C}}$ generated by $\exp \left(\operatorname{adk} \mathfrak{k}_{\mathbb{C}}\right)$. The adjoint group $K_{\mathbb{C}}$ acts canonically on $\mathfrak{p}_{\mathbb{C}}$ and on the dual space $\mathfrak{p}_{\mathbb{C}}^{*}$. Take a Cartan subalgebra $\mathfrak{t}$ of $\mathfrak{k}$ and choose a positive system $\Delta^{+}\left(\mathfrak{k}_{\mathbb{C}}, \mathfrak{t}_{\mathbb{C}}\right)$. Similarly, denote by $\mathfrak{t}^{*}$ the dual space of $\mathfrak{t}$.

Recall the definition for the maximal noncompact root as in [11, Definition 2.1]. If $G$ is not of Hermitian type, then $K_{\mathbb{C}}$ acts irreducibility on $\mathfrak{p}_{\mathbb{C}}$, in which case the maximal noncompact root $\beta \in \sqrt{-1} t^{*}$ is defined to be the highest weight in $\mathfrak{p}_{\mathbb{C}}^{*}$. If $G$ is of Hermitian type, then

$\mathfrak{p}_{\mathbb{C}}=\mathfrak{p}_{+}+\mathfrak{p}_{-}$is an irreducible decomposition as a $K_{\mathbb{C}}$-representation, in which case the maximal noncompact root $\beta \in \sqrt{-1} \mathfrak{t}^{*}$ is defined to be the highest weight in $\mathfrak{p}_{+}^{*}$ where $\mathfrak{p}_{ \pm}^{*}$ is 
the dual space of $\mathfrak{p}_{ \pm}$. Notice that in either case, $-\beta$ is also a weight in $\mathfrak{p}_{\mathbb{C}}^{*}$; in particular, $-\beta$ is a weight in $\mathfrak{p}_{-}^{*}$ for $\mathfrak{g}$ of Hermitian type. The weight spaces $\mathfrak{p}_{\beta}^{*}$ and $\mathfrak{p}_{-\beta}^{*}$ are 1-dimensional.

Let $U\left(\mathfrak{g}_{\mathbb{C}}\right)$ be the universal enveloping algebra of $\mathfrak{g}_{\mathbb{C}}$ with the standard increasing filtration of $\left\{U_{j}\left(\mathfrak{g}_{\mathbb{C}}\right)\right\}_{j \in \mathbb{Z}_{\geq 0}}$. Suppose that $X$ is a finitely generated $\mathfrak{g}_{\mathbb{C}}$-module. A filtration $\left\{X_{i}\right\}_{i \in \mathbb{Z}_{\geq 0}}$ of $X$ is called a good filtration if it satisfies the following conditions:

- $X=\bigcup_{i \in \mathbb{Z} \geq 0} X_{i}$

- $X_{i}$ is a finite dimensional subspace for any $i \in \mathbb{Z}_{\geq 0}$;

- $U_{j}\left(\mathfrak{g}_{\mathbb{C}}\right) X_{i} \subseteq X_{i+j}$ for any $i, j \in \mathbb{Z}_{\geq 0}$;

- there exists $n \in \mathbb{Z}_{\geq 0}$ such that $U_{j}\left(\mathfrak{g}_{\mathbb{C}}\right) X_{i}=X_{i+j}$ for any $i \geq n$ and $j \in \mathbb{Z}_{\geq 0}$.

The graded algebra $\operatorname{gr} U\left(\mathfrak{g}_{\mathbb{C}}\right):=\bigoplus_{j \in \mathbb{Z}_{\geq 0}} U_{j}\left(\mathfrak{g}_{\mathbb{C}}\right) / U_{j-1}\left(\mathfrak{g}_{\mathbb{C}}\right)$ is isomorphic to the symmetric algebra $S\left(\mathfrak{g}_{\mathbb{C}}\right)$, and $\operatorname{gr} X:=\bigoplus_{i \in \mathbb{Z}_{\geq 0}} X_{i} / X_{i-1}$ forms a graded $S\left(\mathfrak{g}_{\mathbb{C}}\right)$-module. Let $\operatorname{Ann}_{S(\mathfrak{g c})}(\operatorname{gr} X):=\{f \in$ $S\left(\mathfrak{g}_{\mathbb{C}}\right) \mid f v=0$ for any $\left.v \in \operatorname{gr} X\right\}$, and the associated variety of $X$ is defined to be $\mathcal{V}_{\mathfrak{g}_{\mathbb{C}}}(X):=$ $\left\{x \in \mathfrak{g}_{\mathbb{C}}^{*} \mid f(x)=0\right.$ for any $\left.f \in \operatorname{Ann}_{S\left(\mathfrak{g}_{\mathbb{C}}\right)}(\operatorname{gr} X)\right\}$, which does not depend on the choice of good filtration.

Let $\mathcal{N}\left(\mathfrak{g}_{\mathbb{C}}^{*}\right)$ be the nilpotent cone of $\mathfrak{g}_{\mathbb{C}}^{*}$, and set $\mathcal{N}\left(\mathfrak{p}_{\mathbb{C}}^{*}\right):=\mathcal{N}\left(\mathfrak{g}_{\mathbb{C}}^{*}\right) \cap \mathfrak{p}_{\mathbb{C}}^{*}$. It is well known that $\mathcal{V}_{\mathfrak{g}_{\mathbb{C}}}(X) \subseteq \mathcal{N}\left(\mathfrak{g}_{\mathbb{C}}^{*}\right)$. Identity $\mathfrak{g}_{\mathbb{C}}^{*}$ with $\mathfrak{g}_{\mathbb{C}}$ by the Killing form, and the adjoint action of $K_{\mathbb{C}}$ on $\mathfrak{g}_{\mathbb{C}}$ induces the coadjoint action on $\mathfrak{g}_{\mathbb{C}}^{*}$.

Proposition 1. Let $G$ be a noncompact connected simple Lie group.

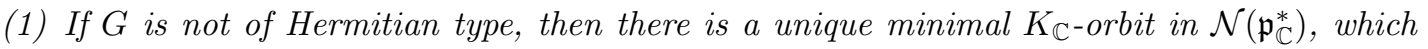
is given by $K_{\mathbb{C}} \cdot\left(\mathfrak{p}_{\beta}^{*} \backslash\{0\}\right)$.

(2) If $G$ is of Hermitian type, then there are two minimal $K_{\mathbb{C}}$-orbits in $\mathcal{N}\left(\mathfrak{p}_{\mathbb{C}}^{*}\right)$, which are given by $K_{\mathbb{C}} \cdot\left(\mathfrak{p}_{\beta}^{*} \backslash\{0\}\right)$ and $K_{\mathbb{C}} \cdot\left(\mathfrak{p}_{-\beta}^{*} \backslash\{0\}\right)$. They have the same dimension.

Proof. See [11, Proposition 2.2].

Remark 2. It is known that the associated variety $\mathcal{V}_{\mathfrak{g}_{\mathbb{C}}}(X)$ is a $K_{\mathbb{C}}$-stable closed subset of $\mathfrak{p}_{\mathbb{C}}^{*}$. Then it follows from Proposition 1 that $K_{\mathbb{C}} \cdot \mathfrak{p}_{\beta}^{*} \subseteq \mathcal{V}_{\mathfrak{g}_{\mathbb{C}}}(X)$ if $G$ is not of Hermitian type, and that $K_{\mathbb{C}} \cdot \mathfrak{p}_{\beta}^{*} \subseteq \mathcal{V}_{\mathfrak{g} \mathbb{C}}(X)$ or $K_{\mathbb{C}} \cdot \mathfrak{p}_{-\beta}^{*} \subseteq \mathcal{V}_{\mathfrak{g} \mathbb{C}}(X)$ if $G$ is of Hermitian type.

2.2. Discrete decomposability for symmetric pairs. Let $G^{\prime}$ be a reductive subgroup of $G$ with the Lie algebra $\mathfrak{g}^{\prime}$. Take a maximal compact subgroup $K$ of $G$, which is defined by a Cartan involution $\theta$ on $G$. In particular, it is assumed that $\theta\left(G^{\prime}\right)=G^{\prime}$; equivalently, $K^{\prime}:=K \cap G^{\prime}$ is a maximal compact subgroup of $G^{\prime}$.

Definition 3. $A(\mathfrak{g}, K)$-module $X$ is called discretely decomposable as a $\left(\mathfrak{g}^{\prime}, K^{\prime}\right)$-module if there exists an increasing filtration $\left\{X_{i}\right\}_{i \in \mathbb{Z}^{+}}$of $\left(\mathfrak{g}^{\prime}, K^{\prime}\right)$-modules such that $\bigcup_{i \in \mathbb{Z}^{+}} X_{i}=X$ and each $X_{i}$ is of finite length as a $\left(\mathfrak{g}^{\prime}, K^{\prime}\right)$-module for any $i \in \mathbb{Z}^{+}$.

Proposition 4. Let $X$ be a unitarizable simple $(\mathfrak{g}, K)$-module. Then the following conditions are equivalent.

(1) $X$ is discretely decomposable as a $\left(\mathfrak{g}^{\Gamma}, K^{\prime}\right)$-module;

(2) there exists a simple $\left(\mathfrak{g}^{\prime}, K^{\prime}\right)$-module $Y$ such that $\operatorname{Hom}_{\left(\mathfrak{g}^{\prime}, K^{\prime}\right)}(Y, X) \neq\{0\}$;

(3) $X$ is isomorphic to an algebraic direct sum of simple $\left(\mathfrak{g}^{\prime}, K^{\prime}\right)$-modules.

Proof. See [9, Lemma 1.3 \& Lemma 1.5]. 
The natural embedding of the Lie algebras $\mathfrak{g}^{\prime} \hookrightarrow \mathfrak{g}$ gives a projection of the complexified dual spaces $\operatorname{pr}_{\mathfrak{g} \rightarrow \mathfrak{g}^{\prime}}: \mathfrak{g}_{\mathbb{C}}^{*} \rightarrow \mathfrak{g}_{\mathbb{C}}^{\prime *}$.

Proposition 5. Let $X$ be a $(\mathfrak{g}, K)$-module of finite length. If $X$ is discretely decomposable as $a\left(\mathfrak{g}^{\prime}, K^{\prime}\right)$-module, then $\operatorname{pr}_{\mathfrak{g} \rightarrow \mathfrak{g}^{\prime}} \mathcal{V}_{\mathbb{C}}(X) \subseteq \mathcal{N}\left(\mathfrak{g}_{\mathbb{C}}^{\prime *}\right)$, where $\mathcal{N}\left(\mathfrak{g}_{\mathbb{C}}^{\prime *}\right)$ is the nilpotent cone of $\mathfrak{g}_{\mathbb{C}}^{\prime *}$.

Proof. See [9, Corollary 3.4].

Now let $\left(G, G^{\sigma}\right)$ be a symmetric pair, where $\sigma \in$ Aut $G$ is an involutive automorphism which commutes with $\theta$. Then $\sigma$ stabilizes $K$, and $K^{\sigma}=K \cap G^{\sigma}$ is a maximal compact subgroup of $G^{\sigma}$. Without confusion, use the same symbol $\sigma$ for its differential on the Lie algebra $\mathfrak{g}$ of $G$, which stabilizes the Lie subalgebra $\mathfrak{k}$ corresponding to $K$. Take a $\sigma$-stable Cartan subalgebra $\mathfrak{t}$ of $\mathfrak{k}$ and a positive root system $\Delta^{+}\left(\mathfrak{k}_{\mathbb{C}}, \mathfrak{t}_{\mathbb{C}}\right)$. Identity $\mathfrak{g}_{\mathbb{C}}^{*}$ with $\mathfrak{g}_{\mathbb{C}}$ by the Killing form, and $\sigma$ can be regarded as an involutive automorphism of $\mathfrak{g}_{\mathbb{C}}^{*}$.

Remember the definition for the maximal noncompact root $\beta$ mentioned in Subsection 2.1.

Proposition 6. Let $G$ be a noncompact connect simple Lie group, and $\left(G, G^{\sigma}\right)$ a symmetric pair. Then there exists a nontrivial unitarizable simple $(\mathfrak{g}, K)$-module $X$ such that $X$ is discretely decomposable as a $\left(\mathfrak{g}^{\sigma}, K^{\sigma}\right)$-module if and only if $\sigma \beta \neq-\beta$.

Proof. See [11, Theorem 5.2 \& Corollary 5.8].

\section{Main Results for Klein four symmetric pairs}

Recall the definition for Klein four symmetric pairs.

Definition 7. Let $G$ (respectively, $\mathfrak{g}$ ) be a real simple Lie group (respectively, Lie algebra), and let $\Gamma$ be a Klein four subgroup of AutG (respectively, Autg). Denote by $G^{\Gamma}$ (respectively, $\mathfrak{g}^{\Gamma}$ ) the subgroup (respectively, subalgebra) of the fixed points under the action of all elements in $\Gamma$ on $G$ (respectively, $\mathfrak{g}$ ). Then $\left(G, G^{\Gamma}\right)$ (respectively, $\left.\left(\mathfrak{g}, \mathfrak{g}^{\Gamma}\right)\right)$ is called a Klein four symmetric pair. In particular, if $G$ (respectively, $\mathfrak{g}$ ) is a real simple Lie group (respectively, Lie algebra) of Hermitian type, and every nonidentity element $\sigma \in \Gamma$ defines a symmetric pair of holomorphic type, then $\left(G, G^{\Gamma}\right)$ (respectively, $\left.\left(\mathfrak{g}, \mathfrak{g}^{\Gamma}\right)\right)$ is called a Klein four symmetric pair of holomorphic type.

For a Klein four symmetric pair $\left(G, G^{\Gamma}\right)$, the maximal compact subgroup $K$ of $G$ is always supposed to be $\Gamma$-stable in the sense that $\sigma(K)=K$ for all $\sigma \in \Gamma$, so that $K^{\Gamma}=K \cap G^{\Gamma}$ is a maximal compact subgroup of $G^{\Gamma}$. Also, fix a $\Gamma$-stable Cartan subalgebra $\mathfrak{t}$ of $\mathfrak{k}$. Similar to the case of symmetric pairs, $\Gamma$ acts on the dual space $\mathfrak{g}_{\mathbb{C}}^{*}$ which is identified with $\mathfrak{g}_{\mathbb{C}}$ by the Killing form.

Proposition 8. If $G$ is a simple Lie group of Hermitian type and $\left(G, G^{\Gamma}\right)$ is a Klein four symmetric pair of holomorphic type, then any irreducible unitary highest / lowest weight $(\mathfrak{g}, K)$ module $X$ is discretely decomposable as a $\left(\mathfrak{g}^{\Gamma}, K^{\Gamma}\right)$-module and is discretely decomposable as a $\left(\mathfrak{g}^{\sigma}, K^{\sigma}\right)$-module for any $\sigma \in \Gamma$.

Proof. The conclusion follows from [8, Theorem 2.9(1) \& Example 2.13 \& Example 3.3] and [9, Proposition 1.6] immediately.

Now consider the discrete decomposability of the restrictions for Klein four symmetric pairs for general cases. Recall Proposition 6 that $\sigma \beta \neq-\beta$ is an equivalent condition for the existence of unitarizable simple $(\mathfrak{g}, K)$-module whose restriction is discretely decomposable for a symmetric pair $\left(G, G^{\sigma}\right)$. The following lemma plays a crucial role in the main result of this article. 
Lemma 9. Let $\left(G, G^{\Gamma}\right)$ be a Klein four symmetric pair. If there are two involutive automorphisms $\sigma$ and $\tau$ in $\Gamma$ such that $\sigma \beta=-\tau \beta \neq \pm \beta$, then $\operatorname{pr}_{\mathfrak{g} \rightarrow \mathfrak{g}^{\Gamma}}\left(K_{\mathbb{C}} \cdot \mathfrak{p}_{\beta}^{*}\right) \nsubseteq \mathcal{N}\left(\mathfrak{g}_{\mathbb{C}}^{\Gamma *}\right)$.

Proof. Obviously, $\sigma \tau \beta=-\beta$ because of $\sigma \beta=-\tau \beta$. Take a nonzero element $x \in \mathfrak{p}_{\beta}^{*}$, and one has $\sigma \tau(x) \in \mathfrak{p}_{-\beta}^{*}$. Let $\bar{x}$ denote the complex conjugation of $x$ with respect to the real form $\mathfrak{g}$ of $\mathfrak{g}_{\mathbb{C}}$, and one also has $\bar{x} \in \mathfrak{p}_{-\beta}^{*}$. Hence, $\overline{\sigma \tau(x)} \in \mathfrak{p}_{\beta}^{*}$ and $y:=x+\overline{\sigma \tau(x)} \in \mathfrak{p}_{\beta}^{*}$. Replace $x$ by $c x$ for some $c \in \mathbb{C}$ if necessary, $y$ can be always assumed to be nonzero. It is known that neither $\sigma(y)$ nor $\tau(y)$ is in $\mathfrak{p}_{ \pm \beta}^{*}$ because $\sigma \beta=-\tau \beta \neq \pm \beta$, and hence $\operatorname{pr}_{\mathfrak{g} \rightarrow \mathfrak{g}^{\Gamma}}(y)=\frac{1}{4}(y+\sigma(y)+\tau(y)+\sigma \tau(y))$ is nonzero. Moreover, one has $\operatorname{pr}_{\mathfrak{g} \rightarrow \mathfrak{g}^{\Gamma}}(y)=\frac{1}{4}(x+\sigma(x)+\tau(x)+\sigma \tau(x)+\bar{x}+\overline{\sigma(x)}+\overline{\tau(x)}+\overline{\sigma \tau(x)}) \in$ $\mathfrak{p}_{\mathbb{C}}^{*} \cap \mathfrak{g}^{*}=\mathfrak{p}^{*}$ which is a nonzero semisimple element. Therefore, $\operatorname{pr}_{\mathfrak{g} \rightarrow \mathfrak{g}^{\Gamma}}(y) \notin \mathcal{N}\left(\mathfrak{g}_{\mathbb{C}}^{\Gamma *}\right)$ and hence $\operatorname{pr}_{\mathfrak{g} \rightarrow \mathfrak{g}^{\Gamma}}\left(K_{\mathbb{C}} \cdot \mathfrak{p}_{\beta}^{*}\right) \nsubseteq \nsubseteq \mathcal{N}\left(\mathfrak{g}_{\mathbb{C}}^{\Gamma *}\right)$.

Remark 10. The condition $\sigma \beta=-\tau \beta \neq \pm \beta$ in Lemma 9 is equivalent to $\sigma(-\beta)=-\tau(-\beta) \neq$ $\pm \beta$, and hence one also obtains that $\operatorname{pr}_{\mathfrak{g} \rightarrow \mathfrak{g}^{\Gamma}}\left(K_{\mathbb{C}} \cdot \mathfrak{p}_{-\beta}^{*}\right) \nsubseteq \mathcal{N}\left(\mathfrak{g}_{\mathbb{C}}^{\Gamma *}\right)$.

The author may state the main theorem now.

Theorem 11. Let $\left(G, G^{\Gamma}\right)$ be a Klein four symmetric pair. If there are two involutive automorphisms $\sigma$ and $\tau$ in $\Gamma$ such that $\sigma \beta=-\tau \beta \neq \pm \beta$, then there does not exist any nontrivial unitarizable simple $(\mathfrak{g}, K)$-module that is discretely decomposable as a $\left(\mathfrak{g}^{\Gamma}, K^{\Gamma}\right)$-module.

Proof. From Remark 2, one has either $K_{\mathbb{C}} \cdot \mathfrak{p}_{\beta}^{*} \subseteq \mathcal{V}_{\mathfrak{g}_{\mathbb{C}}}(X)$ or $K_{\mathbb{C}} \cdot \mathfrak{p}_{-\beta}^{*} \subseteq \mathcal{V}_{\mathfrak{g}_{\mathbb{C}}}(X)$ for any nontrivial unitarizable simple $(\mathfrak{g}, K)$-module $X$. On the other hand, by Lemma 9 and Remark 10, $\operatorname{pr}_{\mathfrak{g} \rightarrow \mathfrak{g}^{\Gamma}}\left(K_{\mathbb{C}} \cdot \mathfrak{p}_{ \pm \beta}^{*}\right) \nsubseteq \mathcal{N}\left(\mathfrak{g}_{\mathbb{C}}^{\Gamma *}\right)$. Therefore, $\operatorname{pr}_{\mathfrak{g} \rightarrow \mathfrak{g}^{\Gamma}} \mathcal{V}_{\mathfrak{g}_{\mathbb{C}}}(X) \nsubseteq \mathcal{N}\left(\mathfrak{g}_{\mathbb{C}}^{\Gamma *}\right)$ for any nontrivial unitarizable simple $(\mathfrak{g}, K)$-module $X$, and the conclusion follows from Proposition 5.

Corollary 12. Let $G$ be a simple Lie group, and $\left(G, G^{\Gamma}\right)$ a Klein four symmetric pair defined by the Klein four subgroup $\Gamma=\langle\sigma, \tau\rangle$ generated by two involutive automorphisms $\sigma$ and $\tau$. Suppose that there exists a nontrivial unitarizable simple $(\mathfrak{g}, K)$-module that is discretely decomposable as a $\left(\mathfrak{g}^{\Gamma}, K^{\Gamma}\right)$-module. Suppose that

(1) there exists a nontrivial unitarizable simple $(\mathfrak{g}, K)$-module that is discretely decomposable as a $\left(\mathfrak{g}^{\sigma}, K^{\sigma}\right)$-module;

(2) there exists a nontrivial unitarizable simple $(\mathfrak{g}, K)$-module that is discretely decomposable as a $\left(\mathfrak{g}^{\tau}, K^{\tau}\right)$-module;

(3) there does not exist any nontrivial unitarizable simple $(\mathfrak{g}, K)$-module that is discretely decomposable as a $\left(\mathfrak{g}^{\sigma \tau}, K^{\sigma \tau}\right)$-module.

Then there does not exist any nontrivial unitarizable simple $(\mathfrak{g}, K)$-module that is discretely decomposable as a $\left(\mathfrak{g}^{\Gamma}, K^{\Gamma}\right)$-module.

Proof. The third condition implies that $\sigma \tau \beta=-\beta$ by Proposition 6 , and hence $\sigma \beta=-\tau \beta$. Again by Proposition 6, the first condition and the second condition imply that $\sigma \beta=-\tau \beta \neq$ $\pm \beta$. The conclusion follows from Theorem 11 .

\section{Applications to $\mathrm{E}_{6(-14)}$}

4.1. Problem and strategy. Let $\left(G, G^{\Gamma}\right)$ be a Klein four symmetric pair. Retain all the settings as previous sections. Consider the following problem.

Problem 13. Classify all the Klein four symmetric pairs $\left(G, G^{\Gamma}\right)$ such that there exists at least one nontrivial unitarizable simple $(\mathfrak{g}, K)$-module $X$ satisfying two conditions: 
- $X$ is discretely decomposable as a $\left(\mathfrak{g}^{\Gamma}, K^{\Gamma}\right)$-module;

- $X$ is discretely decomposable as a $\left(\mathfrak{g}^{\sigma}, K^{\sigma}\right)$-module for some nonidentity element $\sigma \in \Gamma$.

Remark 14. Problem 13 does make sense. In fact, if $X$ is discretely decomposable as a $\left(\mathfrak{g}^{\Gamma}, K^{\Gamma}\right)$-module and $\sigma \in \Gamma$, it is not known whether $X$ is discretely decomposable as a $\left(\mathfrak{g}^{\sigma}, K^{\sigma}\right)$ module. For example, let $G=\operatorname{SL}(n, \mathbb{C})$. Suppose that $\Gamma=\langle\sigma, \tau\rangle$ is the Klein four subgroup generated $\sigma$ and $\tau$, where $\sigma: A \mapsto \bar{A}$ is the natural complex conjugation and $\tau: A \mapsto\left(A^{-1}\right)^{T}$ is the composition of inverse and transposition. Then $G^{\Gamma}=\mathrm{SO}(n)$ is compact and hence any unitarizable simple $(\mathfrak{g}, K)$-module is discretely decomposable as a $\left(\mathfrak{g}^{\Gamma}, K^{\Gamma}\right)$-module, but $G^{\sigma}=\mathrm{SL}(n, \mathbb{R})$ is a split real form of $G$ and by [9, Theorem 8.1], there is no unitarizable simple $(\mathfrak{g}, K)$-module discretely decomposable as a $\left(\mathfrak{g}^{\sigma}, K^{\sigma}\right)$-module.

Remark 15. It is obvious that if $G$ is a simple Lie group of Hermitian type and $\left(G, G^{\Gamma}\right)$ is a Klein four symmetric pair of holomorphic type, then any highest / lowest weight simple $(\mathfrak{g}, K)$-module satisfies the requirements in Problem 13. Moreover, for exceptional Lie groups of Hermitian type, Problem 13 was discussed under the assumption that $\sigma$ is an involutive automorphism of anti-holomorphic type in [3]. In this section, the author considers the case when $\left(G, G^{\Gamma}\right)$ is a Klein four symmetric pair of non-holomorphic type and $\sigma$ is an involutive automorphism of holomorphic type for $G=\mathrm{E}_{6(-14)}$.

The following result is useful to the first step for the classification.

Proposition 16. Let $X$ be a unitarizable simple ( $\mathfrak{g}, K)$-module, and let $\sigma \in \Gamma$. If $X$ is discretely decomposable as a $\left(\mathfrak{g}^{\Gamma}, K^{\Gamma}\right)$-module and $X$ is also discretely decomposable as a $\left(\mathfrak{g}^{\sigma}, K^{\sigma}\right)$ module, then there exists a unitarizable simple $\left(\mathfrak{g}^{\sigma}, K^{\sigma}\right)$-module that is discretely decomposable as a $\left(\mathfrak{g}^{\Gamma}, K^{\Gamma}\right)$-module.

Proof. See [3, Lemma 9].

The author will find out all the Klein four symmetric pairs $\left(G, G^{\Gamma}\right)$ for $G=\mathrm{E}_{6(-14)}$ as required in Problem 13. Since the author excludes the cases when $G^{\Gamma}$ is compact, $\Gamma$ is not supposed to contain any Cartan involution of $G$. Moreover, due to the work in [3], the involutive automorphism $\sigma$ in the second requirement of Problem 13 is supposed to be of holomorphic type, and hence any highest / lowest weight simple $(\mathfrak{g}, K)$-module is discretely decomposable as a $\left(\mathfrak{g}^{\sigma}, K^{\sigma}\right)$-module. Also, because of $\left(G, G^{\Gamma}\right)$ is assumed to be of non-holomorphic type, there is an involutive automorphism $\tau \in \Gamma$, which defines a symmetric pair of anti-holomorphic type. On the other hand, it is known from [5] that, for any nontrivial irreducible unitary representation of $G$, its restriction to its reductive subgroup contains no trivial subrepresentations. Hence in Proposition 16, if $X$ is supposed to be nontrivial, all the direct summands are nontrivial.

Based on the discussion as above, by the conditions in Problem 13 and Proposition 16, the author needs to find reductive Lie group triples $\left(G, G^{\sigma}, G^{\Gamma}\right)$ such that

- $G$ is either $\mathrm{E}_{6(-14)}$;

- $G^{\Gamma}$ is noncompact;

- $\left(G, G^{\sigma}\right)$ is a symmetric pair of holomorphic type;

- $\left(G^{\sigma}, G^{\Gamma}\right)$ is a symmetric pair, such that there exists at least one nontrivial unitarizable simple $\left(\mathfrak{g}^{\sigma}, K^{\sigma}\right)$-module which is discretely decomposable as a $\left(\mathfrak{g}^{\Gamma}, K^{\Gamma}\right)$-module;

- $G^{\Gamma}$ does not contain the center of $K$.

According to [10, Table C.2], there are totally four symmetric pairs $\left(G, G^{\sigma}\right)$ of holomorphic type with $G^{\sigma}$ noncompact, listed in the Lie algebra level $\left(\mathfrak{g}, \mathfrak{g}^{\sigma}\right)$ as follows.

- $\left(\mathfrak{e}_{6(-14)}, \mathfrak{s o}(8,2) \oplus \mathfrak{s o}(2)\right)$

- $\left(\mathfrak{e}_{6(-14)}, \mathfrak{s u}(4,2) \oplus \mathfrak{s u}(2)\right)$ 
- $\left(\mathfrak{e}_{6(-14)}, \mathfrak{s o}^{*}(10) \oplus \mathfrak{s o}(2)\right)$

- $\left(\mathfrak{e}_{6(-14)}, \mathfrak{s u}(5,1) \oplus \mathfrak{s l}(2, \mathbb{R})\right)$

For the time being, the following results ought to be well known and easy to prove.

Proposition 17. Let $\mathfrak{g}=\mathfrak{g}_{1} \oplus \mathfrak{g}_{2}$ be a semisimple Lie algebra that is a direct sum of two nonisomorphic simple ideals $\mathfrak{g}_{1}$ and $\mathfrak{g}_{2}$. If $\varphi$ is a Lie algebra automorphism of $\mathfrak{g}$, then $\varphi \mathfrak{g}_{1}=\mathfrak{g}_{1}$ and $\varphi \mathfrak{g}_{2}=\mathfrak{g}_{2}$.

Proof. It is obvious that $\varphi \mathfrak{g}_{1} \cong \mathfrak{g}_{1}$ is an simple ideal of $\mathfrak{g}$, and hence $\varphi \mathfrak{g}_{1} \cap \mathfrak{g}_{1}$ is an ideal of $\mathfrak{g}$, which is either $\mathfrak{g}_{1}$ or $\{0\}$. Assume $\varphi \mathfrak{g}_{1} \cap \mathfrak{g}_{1}=\{0\}$, and then $\varphi \mathfrak{g}_{1}$ centralizes $\mathfrak{g}_{1}$. But the centralizer of $\mathfrak{g}_{1}$ in $\mathfrak{g}$ is exactly $\mathfrak{g}_{2}$, so $\varphi \mathfrak{g}_{1} \subseteq \mathfrak{g}_{2}$, which contradicts to the simplicity of $\mathfrak{g}_{2}$. Similarly, $\varphi \mathfrak{g}_{2}=\mathfrak{g}_{2}$.

Proposition 18. Let $\mathfrak{g}=Z(\mathfrak{g}) \oplus[\mathfrak{g}, \mathfrak{g}]$ be a reductive Lie algebra with the center $Z(\mathfrak{g})$. If $\varphi$ is a Lie algebra automorphism of $\mathfrak{g}$, then $\varphi Z(\mathfrak{g})=Z(\mathfrak{g})$ and $\varphi[\mathfrak{g}, \mathfrak{g}]=[\mathfrak{g}, \mathfrak{g}]$.

Proof. Any automorphism fixes the center, and then $\varphi Z(\mathfrak{g})=Z(\mathfrak{g})$. Moreover, $\varphi[\mathfrak{g}, \mathfrak{g}]=$ $[\varphi \mathfrak{g}, \varphi \mathfrak{g}]=[\mathfrak{g}, \mathfrak{g}]$.

Suppose that $\mathfrak{g}_{1}$ and $\mathfrak{g}_{2}$ are two simple Lie algebras. It is well known that, if $X_{i}$ is a simple $\left(\mathfrak{g}_{i}, K_{i}\right)$-module for $i=1,2$, then $X_{1} \otimes X_{2}$ is a simple $\left(\mathfrak{g}_{1} \oplus \mathfrak{g}_{2}, K_{1} \times K_{2}\right)$-module. Conversely, any simple $\left(\mathfrak{g}_{1} \oplus \mathfrak{g}_{2}, K_{1} \times K_{2}\right)$-module is in the form of $X_{1} \otimes X_{2}$ where $X_{i}$ is a simple $\left(\mathfrak{g}_{i}, K_{i}\right)$-module for $i=1,2$.

Proposition 19. Let $\mathfrak{g}_{i}$ for $i=1,2$ be two simple Lie algebras, and let $\mathfrak{g}_{i}^{\prime}$ be a reductive subalgebra of $\mathfrak{g}_{i}$. Suppose that $X_{i}$ is a unitarizable simple $\left(\mathfrak{g}_{i}, K_{i}\right)$-module. Then $X_{1} \otimes X_{2}$ is discretely decomposable as a $\left(\mathfrak{g}_{1}^{\prime} \oplus \mathfrak{g}_{2}^{\prime}, K_{1}^{\prime} \times K_{2}^{\prime}\right)$-module if and only if $X_{i}$ is discretely decomposable as a $\left(\mathfrak{g}_{i}^{\prime}, K_{i}^{\prime}\right)$-module for $i=1,2$.

Proof. If $X_{i}$ is discretely decomposable as a $\left(\mathfrak{g}_{i}^{\prime}, K_{i}^{\prime}\right)$-module for $i=1,2$, by Proposition 4 , there exists a simple $\left(\mathfrak{g}_{i}^{\prime}, K_{i}^{\prime}\right)$-module $X_{i}^{\prime}$ such that $\operatorname{Hom}_{\left(\mathfrak{g}_{i}^{\prime}, K_{i}^{\prime}\right)}\left(X_{i}^{\prime}, X_{i}\right) \neq\{0\}$ for $i=1,2$. Take a nonzero element $f_{i} \in \operatorname{Hom}_{\left(\mathfrak{g}_{i}^{\prime}, K_{i}^{\prime}\right)}\left(X_{i}^{\prime}, X_{i}\right)$ for $i=1,2$, and $f_{1} \otimes f_{2}$ is a nonzero element in $\operatorname{Hom}_{\left(\mathfrak{g}_{1}^{\prime} \oplus \mathfrak{g}_{2}^{\prime}, K_{1}^{\prime} \times K_{2}^{\prime}\right)}\left(X_{1}^{\prime} \otimes X_{2}^{\prime}, X_{1} \otimes X_{2}\right)$. Again by Proposition $4, X_{1} \otimes X_{2}$ is discretely decomposable as a $\left(\mathfrak{g}_{1}^{\prime} \oplus \mathfrak{g}_{2}^{\prime}, K_{1}^{\prime} \times K_{2}^{\prime}\right)$-module. Conversely, suppose that $X_{1} \otimes X_{2}$ is discretely decomposable as a $\left(\mathfrak{g}_{1}^{\prime} \oplus \mathfrak{g}_{2}^{\prime}, K_{1}^{\prime} \times K_{2}^{\prime}\right)$-module. By Proposition 4 , there exists a simple $\left(\mathfrak{g}_{i}^{\prime}, K_{i}^{\prime}\right)$ module $X_{i}^{\prime}$ for $i=1,2$ such that $\operatorname{Hom}_{\left(\mathfrak{g}_{1}^{\prime} \oplus \mathfrak{g}_{2}^{\prime}, K_{1}^{\prime} \times K_{2}^{\prime}\right)}\left(X_{1}^{\prime} \otimes X_{2}^{\prime}, X_{1} \otimes X_{2}\right) \neq\{0\}$. Take a nonzero element $v^{\prime} \in X_{2}^{\prime}$. Therefore, as a $\left(\mathfrak{g}_{1}^{\prime}, K_{1}^{\prime}\right)$-module, one has a nonzero injection given by $X_{1}^{\prime} \cong X_{1}^{\prime} \otimes v^{\prime} \hookrightarrow X_{1}^{\prime} \otimes X_{2}^{\prime} \hookrightarrow X_{1} \otimes X_{2} \cong \bigoplus_{v \in X_{2}} X_{1} \otimes v \cong \bigoplus_{v \in X_{2}} X_{1} \hookrightarrow \prod_{v \in X_{2}} X_{1}$. It follows that $\prod_{v \in X_{2}} \operatorname{Hom}_{\left(\mathfrak{g}_{1}^{\prime}, K_{1}^{\prime}\right)}\left(X_{1}^{\prime}, X_{1}\right) \cong \operatorname{Hom}_{\left(\mathfrak{g}_{1}^{\prime}, K_{1}^{\prime}\right)}\left(X_{1}^{\prime}, \prod_{v \in X_{2}} X_{1}\right) \neq\{0\}$, and hence $\operatorname{Hom}_{\left(\mathfrak{g}_{1}^{\prime}, K_{1}^{\prime}\right)}\left(X_{1}^{\prime}, X_{1}\right) \neq$ $\{0\}$. This shows that $X_{1}$ is discretely decomposable as a $\left(\mathfrak{g}_{1}^{\prime}, K_{1}^{\prime}\right)$-module by Proposition 4. Similarly, $X_{2}$ is discretely decomposable as a $\left(\mathfrak{g}_{2}^{\prime}, K_{2}^{\prime}\right)$-module.

Now the author excludes some of the four symmetric pairs $\left(\mathfrak{g}, \mathfrak{g}^{\sigma}\right)$ for $\mathfrak{g}=\mathfrak{e}_{6(-14)}$ listed above. Firstly, let $\left(\mathfrak{g}, \mathfrak{g}^{\sigma}\right)=\left(\mathfrak{e}_{6(-14)}, \mathfrak{s o}^{*}(10) \oplus \mathfrak{s o}(2)\right)$. If $\left(\mathfrak{g}, \mathfrak{g}^{\Gamma}\right)$ is a Klein four symmetric pair, then $\mathfrak{g}^{\Gamma}=\left(\mathfrak{g}^{\sigma}\right)^{\tau}=\mathfrak{s o}^{*}(10)^{\tau} \oplus \mathfrak{s o}(2)^{\tau}$ for some $\tau \in \Gamma$ by Proposition 18. If there is a simple $\left(\mathfrak{g}^{\sigma}, K^{\sigma}\right)$ module that is discretely decomposable as a $\left(\mathfrak{g}^{\Gamma}, K^{\Gamma}\right)$-module, then there exists a simple $\left(\mathfrak{s o}^{*}(10), \mathrm{U}(5)\right)$-module that is discretely decomposable as a $\left(\mathfrak{s o}^{*}(10)^{\tau}, \mathrm{U}(5)^{\tau}\right)$-module by Proposition 19. According to [10, Table C.2] and [11, Theorem 5.2 \& Table 1], such $\left(\mathfrak{s o}^{*}(10), \mathfrak{s o}^{*}(10)^{\tau}\right)$ must be a symmetric pair of holomorphic type; i.e., $\mathfrak{s o}^{*}(10)^{\tau}$ contains the center of $\mathfrak{u}(5)$, the maximal compact subalgebra of $\mathfrak{s o}^{*}(10)$. On the other hand, since $\mathfrak{g}^{\sigma}$ is not compact, the center of $\mathfrak{k}$ cannot centralize the whole $\mathfrak{g}^{\sigma}$. It follows that the center of $\mathfrak{k}$ does not come from $\mathfrak{s o}(2)$, and is equal to the center of $\mathfrak{u}(5)$. Thus, $\mathfrak{g}^{\Gamma}$ contains the center of $\mathfrak{k}$, which 


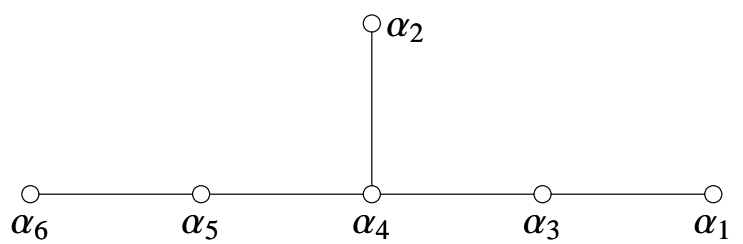

Figure 1. Dynkin diagram of $\mathrm{E}_{6}$.

contradicts to the requirement that $G^{\Gamma}$ does not contain the center of $K$. Hence, there is no need to consider $\left(\mathfrak{g}, \mathfrak{g}^{\sigma}\right)=\left(\mathfrak{e}_{6(-14)}, \mathfrak{s o}^{*}(10) \oplus \mathfrak{s o}(2)\right)$.

Secondly, let $\left(\mathfrak{g}, \mathfrak{g}^{\sigma}\right)=\left(\mathfrak{g}, \mathfrak{g}_{1} \oplus \mathfrak{g}_{2}\right)=\left(\mathfrak{e}_{6(-14)}, \mathfrak{s u}(5,1) \oplus \mathfrak{s}(2, \mathbb{R})\right)$. If $\left(\mathfrak{g}, \mathfrak{g}^{\Gamma}\right)$ is a Klein four symmetric pair, by Proposition $17, \mathfrak{g}^{\Gamma}=\left(\mathfrak{g}^{\sigma}\right)^{\tau}=\mathfrak{g}_{1}^{\tau} \oplus \mathfrak{g}_{2}^{\tau}$ for some $\tau \in \Gamma$. If $X_{1} \otimes X_{2}$ is a simple $\left(\mathfrak{g}^{\sigma}, K^{\sigma}\right)$ module that is discretely decomposable as a $\left(\mathfrak{g}^{\Gamma}, K^{\Gamma}\right)$-module, $X_{i}$ is discretely decomposable as a $\left(\mathfrak{g}_{i}^{\tau}, K_{i}^{\tau}\right)$-module by Proposition 19. According to [10, Table C.2] and [11, Theorem $5.2 \&$ Table 1], for $\mathfrak{g}_{1}=\mathfrak{s u}(5,1)$ or $\mathfrak{g}_{2}=\mathfrak{s l}(2, \mathbb{R})$, if there exists a simple $\left(\mathfrak{g}_{i}, K_{i}\right)$ module that is discretely decomposable as a $\left(\mathfrak{g}_{i}^{\tau}, K_{i}^{\tau}\right)$-module, $\left(\mathfrak{g}_{i}, \mathfrak{g}_{i}^{\tau}\right)$ must be a symmetric pair of holomorphic type. Hence, $\mathfrak{g}^{\Gamma}$ contains the center of $\mathfrak{k}^{\sigma}$. Since $\left(\mathfrak{g}, \mathfrak{g}^{\sigma}\right)$ is supposed to be of holomorphic type, the center of $\mathfrak{k}^{\sigma}$ contains that of $\mathfrak{k}$. But this does not satisfy the requirement that $G^{\Gamma}$ does not contain the center of $K$. Hence, there is no need to consider $\left(\mathfrak{g}, \mathfrak{g}^{\sigma}\right)=\left(\mathfrak{e}_{6(-14)}, \mathfrak{s u}(5,1) \oplus \mathfrak{s l}(2, \mathbb{R})\right)$.

Thus, there remain two symmetric pairs of holomorphic type $\left(\mathfrak{g}, \mathfrak{g}^{\sigma}\right)$.

- $\left(\mathfrak{e}_{6(-14)}, \mathfrak{s o}(8,2) \oplus \mathfrak{s o}(2)\right)$

- $\left(\mathfrak{e}_{6(-14)}, \mathfrak{s u}(4,2) \oplus \mathfrak{s u}(2)\right)$

By the similar argument as in the case of $\left(\mathfrak{e}_{6(-14)}, \mathfrak{s 0 ^ { * }}(10) \oplus \mathfrak{s o}(2)\right)$, the center of the maximal compact subalgebra of $\mathfrak{e}_{6(-14)}$ is contained in $\mathfrak{s o}(8,2)$ and $\mathfrak{s u}(4,2)$ for $\left(\mathfrak{g}, \mathfrak{g}^{\sigma}\right)=\left(\mathfrak{e}_{6(-14)}, \mathfrak{s o}(8,2) \oplus\right.$ $\mathfrak{s o}(2))$ and $\left(\mathfrak{e}_{6(-14)}, \mathfrak{s u}(4,2) \oplus \mathfrak{s u}(2)\right)$ respectively. Notice that $\left(\mathfrak{g}^{\sigma}, \mathfrak{g}^{\Gamma}\right)$ is a symmetric pair and $\mathfrak{g}^{\Gamma}$ does not contain the center of $\mathfrak{k}$. By Proposition 17, Proposition 18, [10, Table C.2], and $\left[11\right.$, Table 1], the possible triples $\left(\mathfrak{g}, \mathfrak{g}^{\sigma}, \mathfrak{g}^{\Gamma}\right)$ are

$$
\begin{array}{ll}
\left(\mathfrak{e}_{6(-14)}, \mathfrak{s o}(8,2) \oplus \mathfrak{s o}(2), \mathfrak{s o}(8,1) \oplus \mathfrak{s o}(2)\right), & \left(\mathfrak{e}_{6(-14)}, \mathfrak{s o}(8,2) \oplus \mathfrak{s o}(2), \mathfrak{s o}(8,1)\right) ; \\
\left(\mathfrak{e}_{6(-14)}, \mathfrak{s u}(4,2) \oplus \mathfrak{s u}(2), \mathfrak{s p}(2,1) \oplus \mathfrak{s u}(2)\right), & \left(\mathfrak{e}_{6(-14)}, \mathfrak{s u}(4,2) \oplus \mathfrak{s u}(2), \mathfrak{s p}(2,1) \oplus \mathfrak{s o}(2)\right) .
\end{array}
$$

The author does not need to consider $\left(\mathfrak{e}_{6(-14)}, \mathfrak{s o}(8,2) \oplus \mathfrak{s o}(2), \mathfrak{s o}(8,1)\right)$ because of the following result.

Proposition 20. For $\left(\mathfrak{g}, \mathfrak{g}^{\sigma}, \mathfrak{g}^{\Gamma}\right)=\left(\mathfrak{e}_{6(-14)}, \mathfrak{s o}(8,2) \oplus \mathfrak{s o}(2), \mathfrak{s o}(8,1)\right)$, $\left(\mathfrak{g}, \mathfrak{g}^{\Gamma}\right)$ is a Klein four symmetric pair. Moreover, there exists a unitarizable simple $(\mathfrak{g}, K)$-module that is discretely decomposable as a $\left(\mathfrak{g}^{\sigma}, K^{\sigma}\right)$-module and is discretely decomposable as a $\left(\mathfrak{g}^{\Gamma}, K^{\Gamma}\right)$-module.

Proof. See [3, Proposition 10 \& Proposition 13].

In the next two subsections, the author will determine whether each of the remaining there pairs $\left(\mathfrak{g}, \mathfrak{g}^{\Gamma}\right)$ is really a Klein four symmetric pair.

4.2. Elementary abelian 2-subgroups of Aute $\mathfrak{e}_{6(-78)}$. Let $\mathfrak{e}_{6}$ be the complex simple Lie algebra of type $\mathrm{E}_{6}$. Fix a Cartan subalgebra of $\mathfrak{e}_{6}$ and a simple root system $\left\{\alpha_{i} \mid 1 \leq i \leq 6\right\}$, the Dynkin diagram of which is given in Figure 1. For each root $\alpha$, denote by $H_{\alpha}$ its coroot, and denote by $X_{\alpha}$ the normalized root vector so that $\left[X_{\alpha}, X_{-\alpha}\right]=H_{\alpha}$. Moreover, one can normalize $X_{\alpha}$ appropriately such that

$$
\operatorname{Span}_{\mathbb{R}}\left\{X_{\alpha}-X_{-\alpha}, \sqrt{-1}\left(X_{\alpha}+X_{-\alpha}\right), \sqrt{-1} H_{\alpha} \mid \alpha: \text { positive root }\right\} \cong \mathfrak{e}_{6(-78)}
$$


is a compact real form of $\mathfrak{e}_{6}$ by [6]. It is well known that

$$
\operatorname{Aute}_{6(-78)} / \operatorname{Int}_{6(-78)} \cong \operatorname{Aute}_{6} / \operatorname{Int}_{6}
$$

which is just the automorphism group of the Dynkin diagram.

Follow the constructions of involutive automorphisms of $\mathfrak{e}_{6(-78)}$ in [12]. Let $\omega$ be the specific involutive automorphism of the Dynkin diagram defined by

$$
\begin{array}{ll}
\omega\left(H_{\alpha_{1}}\right)=H_{\alpha_{6}}, & \omega\left(X_{ \pm \alpha_{1}}\right)=X_{ \pm \alpha_{6}}, \\
\omega\left(H_{\alpha_{2}}\right)=H_{\alpha_{2}}, & \omega\left(X_{ \pm \alpha_{2}}\right)=X_{ \pm \alpha_{2}}, \\
\omega\left(H_{\alpha_{3}}\right)=H_{\alpha_{5}}, & \omega\left(X_{ \pm \alpha_{3}}\right)=X_{ \pm \alpha_{5}}, \\
\omega\left(H_{\alpha_{4}}\right)=H_{\alpha_{4}}, & \omega\left(X_{ \pm \alpha_{4}}\right)=X_{ \pm \alpha_{4}} .
\end{array}
$$

Let $\sigma_{1}=\exp \left(\sqrt{-1} \pi H_{\alpha_{2}}\right), \sigma_{2}=\exp \left(\sqrt{-1} \pi\left(H_{\alpha_{1}}+H_{\alpha_{6}}\right)\right), \sigma_{3}=\omega, \sigma_{4}=\omega \exp \left(\sqrt{-1} \pi H_{\alpha_{2}}\right)$, where exp represents the exponential map from $\mathfrak{e}_{6(-78)}$ to Aute $6(-78)$. Then $\sigma_{1}, \sigma_{2}, \sigma_{3}$, and $\sigma_{4}$ represent all conjugacy classes of involutive automorphisms in Aute $\mathfrak{e}_{6(-78)}$, which correspond to real forms $\mathfrak{e}_{6(2)}, \mathfrak{e}_{6(-14)}, \mathfrak{e}_{6(-26)}$, and $\mathfrak{e}_{6(6)}$ respectively.

From [12], it is known that $\left(\operatorname{Inte}_{6(-78)}\right)^{\sigma_{3}} \cong \mathrm{F}_{4(-52)}$, the compact Lie group of type $\mathrm{F}_{4}$, and there exist involutive automorphisms $\tau_{1}$ and $\tau_{2}$ of $\mathrm{F}_{4(-52)}$ such that $\mathfrak{f}_{4(-52)}^{\tau_{1}} \cong \mathfrak{s p}(3) \oplus \mathfrak{s p}(1)$ and $\mathfrak{f}_{4(-52)}^{\tau_{2}} \cong \mathfrak{s o}(9)$, where $\mathfrak{f}_{4(-52)}$ denotes the compact Lie algebra of type $\mathrm{F}_{4}$. Moreover, $\tau_{1}$ and $\tau_{2}$ represent all conjugacy classes of involutive automorphisms in Autf $f_{4(-52)}$. Now, consider $\left(\left(\operatorname{Inte}_{6(-78)}\right)^{\sigma_{3}}\right)^{\tau_{1}} \cong \operatorname{Sp}(3) \times \operatorname{Sp}(1) /\left\langle\left(-I_{3},-1\right)\right\rangle$. Let $\mathbf{i}, \mathbf{j}$, and $\mathbf{k}$ denote the fundamental quaternion units, and then set $x_{0}=\sigma_{3}, x_{1}=\tau_{1}=\left(I_{3},-1\right), x_{2}=\left(\mathbf{i} I_{3}, \mathbf{i}\right), x_{3}=\left(\mathbf{j} I_{3}, \mathbf{j}\right)$, $x_{4}=\left(\left(\begin{array}{ccc}-1 & 0 & 0 \\ 0 & -1 & 0 \\ 0 & 0 & 1\end{array}\right), 1\right)$, and $x_{5}=\left(\left(\begin{array}{ccc}-1 & 0 & 0 \\ 0 & 1 & 0 \\ 0 & 0 & -1\end{array}\right), 1\right)$.

For a pair $(r, s)$ of integers with $r \leq 2$ and $s \leq 3$, define

$$
F_{r, s}:=\left\langle x_{0}, x_{1}, \cdots, x_{s}, x_{4}, x_{5}, \cdots, x_{r+3}\right\rangle
$$

and

$$
F_{r, s}^{\prime}:=\left\langle x_{1}, x_{2}, \cdots, x_{s}, x_{4}, x_{5}, \cdots, x_{r+3}\right\rangle .
$$

Remark 21. Besides the two families $F_{r, s}$ and $F_{r, s}^{\prime}$ of the elementary abelian 2-subgroups for Aute $_{6(-78)}$ through $\sigma_{3}$, Jun $Y U$ also defined another two families $F_{u, v, r, s}$ and $F_{u, v, r, s}^{\prime}$ through $\sigma_{4}$ in [12]. According to [12, Proposition 6.3 \& Proposition 6.5], each elementary abelian 2subgroup in Aute ${ }_{6(-78)}$ is conjugate to one of the groups in the one family of $F_{r, s}, F_{r, s}^{\prime}, F_{u, v, r, s}$, and $F_{u, v, r, s}^{\prime}$, and all groups in the families of $F_{r, s}, F_{r, s}^{\prime}, F_{u, v, r, s}$, and $F_{u, v, r, s}^{\prime}$ are pairwisely nonconjugate. In this article, $F_{u, v, r, s}$ and $F_{u, v, r, s}^{\prime}$ will not be used, so the author does not write down the detailed constructions for them.

4.3. Klein four symmetric pairs for $\mathfrak{e}_{6(-14)}$. If $\sigma$ is an involutive automorphism of $\mathfrak{e}_{6(-78)}$, which is conjugate to $\sigma_{2}$ as defined above, then by the construction, $\mathfrak{e}_{6(-14)} \cong \mathfrak{e}_{6(-78)}^{\sigma}+$ $\sqrt{-1} \mathfrak{e}_{6(-78)}^{-\sigma}$, where $\mathfrak{e}_{6(-78)}^{ \pm \sigma}$ are the eigenspaces of eigenvalues \pm 1 under the action of $\sigma$ on $\mathfrak{e}_{6(-78)}$. Thus, if $\left\langle\sigma, \mu_{1}, \mu_{2}, \cdots, \mu_{n}\right\rangle$ is an elementary abelian 2-subgroups of rank $n+1$ of Aute $_{6(-78)}$, then $\left\langle\mu_{1}, \mu_{2}, \cdots, \mu_{n}\right\rangle$ is an elementary abelian 2-subgroups of rank $n$ of Aute $6(-14)$. Conversely, if $\left\langle\eta_{1}, \eta_{2}, \cdots, \eta_{n}\right\rangle$ is an elementary abelian 2-subgroups of rank $n$ of Aute $6(-14)$, by $\left[1\right.$, Proposition 1] there exists a Cartan involution $\sigma \in \operatorname{Aute}_{6(-14)}$ such that $\left\langle\sigma, \eta_{1}, \eta_{2}, \cdots, \eta_{n}\right\rangle$ is an elementary abelian 2-subgroups of rank $n+1$ of Aute $6(-78)$. Under this correspondence, $\mathfrak{e}_{6(-78)}^{\left\langle\eta_{1}, \eta_{2}, \cdots, \eta_{n}\right\rangle}$ is the compact dual of $\mathfrak{e}_{6(-14)}^{\left\langle\eta_{1}, \eta_{2}, \cdots, \eta_{n}\right\rangle}$ whose maximal compact subalgebra is $\mathfrak{e}_{6(-14)}^{\left\langle\sigma, \eta_{1}, \eta_{2}, \cdots, \eta_{n}\right\rangle} \cong \mathfrak{e}_{6(-78)}^{\left\langle\sigma, \eta_{1}, \eta_{2}, \cdots, \eta_{n}\right\rangle}$.

Lemma 22. The pair $\left(\mathfrak{e}_{6(-14)}, \mathfrak{s p}(2,1) \oplus \mathfrak{s u}(2)\right)$ is a Klein four symmetric pair, while neither $\left(\mathfrak{e}_{6(-14)}, \mathfrak{s o}(8,1) \oplus \mathfrak{s o}(2)\right)$ nor $\left(\mathfrak{e}_{6(-14)}, \mathfrak{s p}(2,1) \oplus \mathfrak{s o}(2)\right)$ is. 
Proof. Retain all the notations as in Section 4.2. For the first statement, consider the subgroup $F_{1,1}=\left\langle x_{0}, x_{1}, x_{4}\right\rangle$ of Aute $_{6(-78)}$. It is known from [12] and [1, Lemma 8] that $x_{0}$ is conjugate to $\sigma_{3}, x_{1}$ is conjugate to $\sigma_{1}$, and $x_{4}$ is conjugate to $\sigma_{2}$. Then $\mathfrak{e}_{6(-14)} \cong \mathfrak{e}_{6(-78)}^{x_{4}}+\sqrt{-1} \mathfrak{e}_{6(-78)}^{-x_{4}}$. According to [12, Table 3], up to conjugation, the only Klein four subgroup of Aute $\mathfrak{e}_{6(-78)}$, which contains both an element conjugate to $\sigma_{1}$ and an element conjugate to $\sigma_{3}$, is $\Gamma_{5}$ (using the notation in [12, Table 3]), so $\mathfrak{e}_{6(-78)}^{\left\langle x_{0}, x_{1}\right\rangle} \cong \mathfrak{e}_{6(-78)}^{\Gamma_{5}} \cong \mathfrak{s p}(3) \oplus \mathfrak{s u}(2)$ is the compact dual of $\mathfrak{e}_{6(-14)}^{\left\langle x_{0}, x_{1}\right\rangle}$. On the other hand, $\mathfrak{e}_{6(-14)}^{\left\langle x_{0}, x_{1}, x_{4}\right\rangle}=\mathfrak{e}_{6(-78)}^{\left\langle x_{0}, x_{1}, x_{4}\right\rangle}$ is the maximal compact subalgebra of $\mathfrak{e}_{6(-14)}^{\left\langle x_{0}, x_{1}\right\rangle}$. Notice that $\mathfrak{e}_{6(-78)}^{x_{0}} \cong \mathfrak{f}_{4(-52)}$. Moreover, $x_{1}$ is conjugate to $\tau_{1}$ and $x_{4}$ is conjugate to $\tau_{2}$ in Autf $\mathfrak{f}_{4(-52)}$. Again by [12, Table 3], up to conjugation, the only Klein four subgroup of Autf (1(-52) $_{2}$, which contains both an element conjugate to $\tau_{1}$ and an element conjugate to $\tau_{2}$, is $\Gamma_{2}$ (using the notation in [12, Table 3]), so $\mathfrak{e}_{6(-14)}^{\left\langle x_{0}, x_{1}, x_{4}\right\rangle}=\left(\mathfrak{e}_{6(-78)}^{x_{0}}\right)^{\left\langle x_{1}, x_{4}\right\rangle} \cong \mathfrak{f}_{4(-52)}^{\left\langle x_{1}, x_{4}\right\rangle} \cong \mathfrak{f}_{4(-52)}^{\Gamma_{2}} \cong \mathfrak{s o}(5) \oplus 2 \mathfrak{s u}(2)$ is the maximal compact subalgebra of $\mathfrak{e}_{6(-14)}^{\left\langle x_{0}, x_{1}\right\rangle}$. Thus, one obtains $\mathfrak{e}_{6(-14)}^{\left\langle x_{0}, x_{1}\right\rangle} \cong \mathfrak{s p}(2,1) \oplus \mathfrak{s u}(2)$. For the second statement, it follows immediately from the fact that there is no Klein four subgroups $\Gamma \subseteq \operatorname{Aute}_{6(-78)}$ such that $\mathfrak{e}_{6(-78)}^{\Gamma}$ is conjugate to $\mathfrak{s o}(9) \oplus \mathfrak{s o}(2)$ or $\mathfrak{s p}(3) \oplus \mathfrak{s o}(2)$, the compact dual of $\mathfrak{s o}(8,1) \oplus \mathfrak{s o}(2)$ or $\mathfrak{s p}(2,1) \oplus \mathfrak{s o}(2)$, according to [12, Table 3].

4.4. Discrete decomposability for $\left(\mathfrak{e}_{6(-14)}, \mathfrak{s p}(2,1) \oplus \mathfrak{s u}(2)\right)$. In the final subsection, the author focuses on the Klein four symmetric pair $\left(\mathfrak{e}_{6(-14)}, \mathfrak{s p}(2,1) \oplus \mathfrak{s u}(2)\right)$. In order to apply Theorem 11 or Corollary 12 to the pair, the author needs to know how each nonidentity element behaves on $\mathfrak{e}_{6(-14)}$. Retain the notations as in Subsection 4.2, it is known from the proof for Lemma 22 that $\mathfrak{e}_{6(-14)} \cong \mathfrak{e}_{6(-78)}^{x_{4}}+\sqrt{-1} \mathfrak{e}_{6(-78)}^{-x_{4}}$ and $\Gamma=\left\langle x_{0}, x_{1}\right\rangle$.

Lemma 23. One has $\mathfrak{r}_{6(-14)}^{x_{0}} \cong \mathfrak{f}_{4(-20)}, \mathfrak{e}_{6(-14)}^{x_{1}} \cong \mathfrak{s u}(4,2) \oplus \mathfrak{s u}(2)$, and $\mathfrak{e}_{6(-14)}^{x_{0} x_{1}} \cong \mathfrak{s p}(2,2)$.

Proof. Since $x_{0}$ is conjugate to $\sigma_{3}$ in Aute $\mathfrak{e}_{6(-14)}$, it follows from [12, Table 2] that $\mathfrak{e}_{6(-78)}^{x_{0}} \cong$ $\mathfrak{f}_{4(-52)}$, which is the compact dual of $\mathfrak{e}_{6(-14)}^{x_{0}}$. Moreover, since $x_{4}$ is conjugate to $\sigma_{2}$, according to [12, Table 3], up to conjugation the only Klein four subgroup of Aute $\mathfrak{e}_{6(-14)}$, which contains both an element conjugate to $\sigma_{2}$ and an element conjugate to $\sigma_{3}$, is $\Gamma_{7}$ (using the notation in [12, Table 3]). Thus, $\mathfrak{e}_{6(-14)}^{\left\langle x_{0}, x_{4}\right\rangle} \cong \mathfrak{s o}(9)$ which is the maximal compact subalgebra of $\mathfrak{e}_{6(-14)}^{x_{0}}$. Therefore, $\mathfrak{e}_{6(-14)}^{x_{0}} \cong \mathfrak{f}_{4(-20)}$.

Since $x_{1}$ is conjugate to $\sigma_{1}$ in Aute $\mathfrak{e}_{6(-14)}$, it follows from [12, Table 2] that $\mathfrak{e}_{6(-78)}^{x_{1}} \cong \mathfrak{s u}(6) \oplus$ $\mathfrak{s u}(2)$, which is the compact dual of $\mathfrak{e}_{6(-14)}^{x_{1}}$. Moreover, since $x_{4}$ is conjugate to $\sigma_{2}$ and $x_{1} x_{4}$ is conjugate to $\sigma_{1}$ in $\operatorname{Aute}_{6(-14)}$ by [1, Lemma 8], according to [12, Table 3], $\mathfrak{e}_{6(-14)}^{\left\langle x_{1}, x_{4}\right\rangle}=\mathfrak{e}_{6(-78)}^{\left\langle x_{1}, x_{4}\right\rangle} \cong$ $\mathfrak{s u}(4) \oplus 2 \mathfrak{s u}(2) \oplus \mathfrak{s o}(2)$ which is the maximal compact subalgebra of $\mathfrak{e}_{6(-14)}^{x_{1}}$. Therefore, $\mathfrak{e}_{6(-14)}^{x_{1}} \cong$ $\mathfrak{s u}(4,2) \oplus \mathfrak{s u}(2)$.

It is known from [12] that $x_{0} x_{1}=\sigma_{3} \tau_{1}$ is conjugate to $\sigma_{4}$, and hence $\mathfrak{e}_{6(-78)}^{x_{0} x_{1}} \cong \mathfrak{s p}(4)$ by [12, Table 2], which is the compact dual of $\mathfrak{e}_{6(-14)}^{x_{0} x_{1}}$. Moreover, since $x_{4}$ is conjugate to $\sigma_{2}$, according to [12, Table 3], up to conjugation the only Klein four subgroup of Aute $\mathbf{e}_{6(-14)}$, which contains both an element conjugate to $\sigma_{2}$ and an element conjugate to $\sigma_{4}$, is $\Gamma_{8}$ (using the notation in $\left[12\right.$, Table 3]). Thus, $\mathfrak{e}_{6(-14)}^{\left\langle x_{0} x_{1}, x_{4}\right\rangle} \cong 2 \mathfrak{s o}(5) \cong 2 \mathfrak{s p}(2)$ which is the maximal compact subalgebra of $\mathfrak{e}_{6(-14)}^{x_{0}}$. Therefore, $\mathfrak{e}_{6(-14)}^{x_{0} x_{1}} \cong \mathfrak{s p}(2,2)$.

Lemma 24. There does not exist any nontrivial unitarizable simple $\left(\mathfrak{e}_{6(-14)}, \mathrm{SO}(10) \times \mathrm{SO}(2)\right)$ module that is discretely decomposable as a $\left(\mathfrak{s p}(2,1) \oplus \mathfrak{s u}(2), \mathrm{Sp}(2) \times \mathrm{SU}(2)^{2}\right)$-module.

Proof. It is known from [11, Theorem $5.2 \&$ Corollary $5.8 \&$ Table 1] that there exists a nontrivial unitarizable simple $\left(\mathfrak{e}_{6(-14)}, \mathrm{SO}(10) \times \mathrm{SO}(2)\right)$-module which is discretely decomposable as a $\left(\mathfrak{f}_{4(-20)}, \mathfrak{s o}(9)\right)$-module, that there exists a nontrivial unitarizable simple $\left(\mathfrak{e}_{6(-14)}, \mathrm{SO}(10) \times\right.$ 
$\mathrm{SO}(2))$-module which is discretely decomposable as a $\left(\mathfrak{s u}(4,2) \oplus \mathfrak{s u}(2), \mathrm{SU}(2)^{2} \times \mathrm{SO}(2)\right)$-module, and that there does not exist any nontrivial unitarizable simple $\left(\mathfrak{e}_{6(-14)}, \mathrm{SO}(10) \times \mathrm{SO}(2)\right)$ module which is discretely decomposable as a $\left(\mathfrak{s p}(2,2), \mathrm{Sp}(2)^{2}\right)$-module. Therefore, the conclusion follows from Lemma 23 and Corollary 12.

Now the author can solve Problem 13 for $G=\mathrm{E}_{6(-14)}$.

Theorem 25. Let $G=\mathrm{E}_{6(-14)}$ and $\left(G, G^{\Gamma}\right)$ a Klein four symmetric pair with $G^{\Gamma}$ noncompact. Then there exists a nontrivial unitarizable simple $(\mathfrak{g}, K)$-module $X$ that is discretely decomposable as a $\left(\mathfrak{g}^{\Gamma}, K^{\Gamma}\right)$-module and is also discretely decomposable as a $\left(\mathfrak{g}^{\sigma}, K^{\sigma}\right)$-module for some nonidentity element $\sigma \in \Gamma$, if and only if $\left(\mathfrak{g}, \mathfrak{g}^{\Gamma}\right)=\left(\mathfrak{e}_{6(-14)}, \mathfrak{s o}(8,1)\right)$.

Proof. If $\sigma$ is such that $\left(\mathfrak{g}, \mathfrak{g}^{\sigma}\right)$ is a symmetric pair of holomorphic type, the conclusion follows from Proposition 20 and Lemma 24. If $\sigma$ is such that $\left(\mathfrak{g}, \mathfrak{g}^{\sigma}\right)$ is a symmetric pair of antiholomorphic type, the conclusion follows from [3, Proposition 13 \& Proposition 15].

\section{REFERENCES}

[1] Haian HE, Classification of Klein four symmetric pairs of holomorphic type for $\mathrm{E}_{6(-14)}$, Geometriae Dedicata, Volume 197 (2018), Page 77-89.

[2] Haian HE, Classification of Klein four symmetric pairs of holomorphic type for $\mathrm{E}_{7(-25)}$, Geometriae Dedicata, Volume 202 (2019), Page 153-164.

[3] Haian HE, Discretely decomposable restrictions of $(\mathfrak{g}, K)$-modules for Klein four symmetric pairs of exceptional Lie groups of Hermitian type, International Journal of Mathematics, DOI: 10.1142/S0129167X20500019.

[4] Jingsong HUANG and Jun YU, Klein four-subgroups of Lie algebra automorphisms, Pacific Journal of Mathematics, Volume 262 (2013), Number 2, Page 1-100.

[5] Roger Evans HOWE and Calvin Cooper MOORE, Asymptotic properties of unitary representations, Journal of Functional Analysis, Volume 32 (1979), Number 1, Page 72-96.

[6] Anthony William KNAPP, Lie groups beyond an introduction (Second Edition), Progress in Mathematics, Volume 140 (2002), Birkhäuser Boston·Basel·Berlin.

[7] Toshiyuki KOBAYASHI, Discrete decomposability of the restriction of $A_{\mathfrak{q}}(\lambda)$ with respect to reductive subgroups and its applications, Inventiones Mathematicae, Volume 117 (1994), Number 2, Page 181-205.

[8] Toshiyuki KOBAYASHI, Discrete decomposability of the restriction of $A_{\mathfrak{q}}(\lambda)$ with respect to reductive subgroups II: micro-local analysis and asymptotic K-support, Annals of Mathematics (Second Series), Volume 147 (1998), Number 3, Page 709-729.

[9] Toshiyuki KOBAYASHI, Discrete decomposability of the restriction of $A_{\mathfrak{q}}(\lambda)$ with respect to reductive subgroups III: restriction of Harish-Chandra modules and associated varieties, Inventiones Mathematicae, Volume 131 (1998), Page 229-256.

[10] Toshiyuki KOBAYASHI and Yoshiki OSHIMA, Classification of discretely decomposable $A_{\mathfrak{q}}(\lambda)$ with respect to reductive symmetric pairs, Advances in Mathematics, Volume 231 (2012), Number 3-4, Page 2013-2047.

[11] Toshiyuki KOBAYASHI and Yoshiki OSHIMA, Classification of symmetric pairs with discretely decomposable restrictions of $(\mathfrak{g}, K)$-modules, Journal für die Reine und Angewandte Mathematik, Volume 703 (2015), Page 201-223.

[12] Jun YU, Elementary abelian 2-subgroups of compact Lie groups, Geometriae Dedicata, Volume 167 (2013), Page 245-293.

Department of Mathematics, College of Sciences, Shanghai University, No. 99 Shangda Road, Baoshan District, Shanghai, 200444 China P. R.

E-mail address: hebe.hsinchu@yahoo.com.tw 